Editorial

\title{
International Archives of Otorhinolaryngology is now Indexed in Emerging Sources Citation Index of Web of Science
}

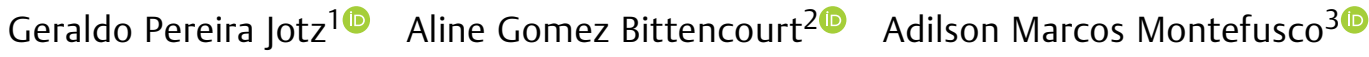

${ }^{1}$ Department of Morphological Sciences, Universidade Federal do Rio Grande do Sul, Porto Alegre, RS, Brazil

${ }^{2}$ Department of Otorhinolaryngology, Universidade Federal do Maranhão, São Luís, MA, Brazil

${ }^{3}$ Department of Otorhinolaryngology, Universidade de São Paulo, São Paulo, SP, Brazil

Int Arch Otorhinolaryngol 2020;24:e1-e4.

We are pleased to announce that the International Archives of Otorhinolaryngology (IAO) is now indexed in the Emerging Sources Citation Index (ESCI) of the Web of Science (WoS) Core Collection. ESCI is a multidisciplinary citation index that includes peer-reviewed journals that follow ethi- cal publishing practices and meet the WoS' stringent technical requirements. ${ }^{1,2}$

Inclusion in $\mathrm{ESCl}$ is an important milestone for the IAO. It enables authors and readers to follow and track the number of citations received by the WoS in real time (-Figs. 1-2). In

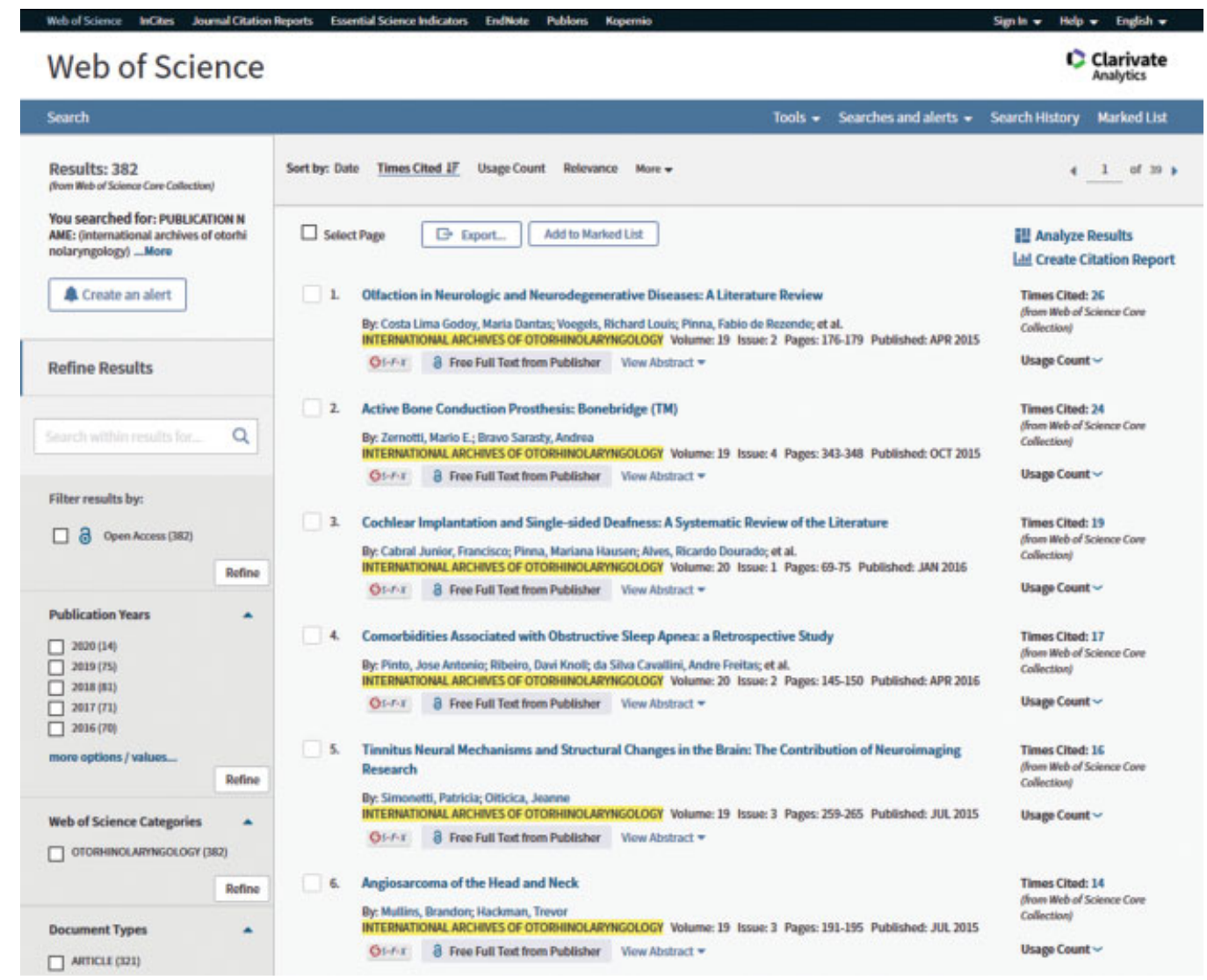

Fig. 1 Citation report by article in the Web of Science.

Address for correspondence Prof. Geraldo Pereira Jotz, MD, PhD, Department of Morphological Sciences, Universidade Federal do Rio Grande do Sul, Avenida Paulo Gama 110-Farroupilha, Porto Alegre, RS, 90040-060, Brazil (e-mail: iaorl@iaorl.org).
DOI https://doi.org/ 10.1055/s-0039-3402452. ISSN 1809-9777.
Copyright @ 2020 by Thieme Revinter Publicações Ltda, Rio de Janeiro, Brazil
License terms

(c) (i) $\ominus$ (ㅇ) 


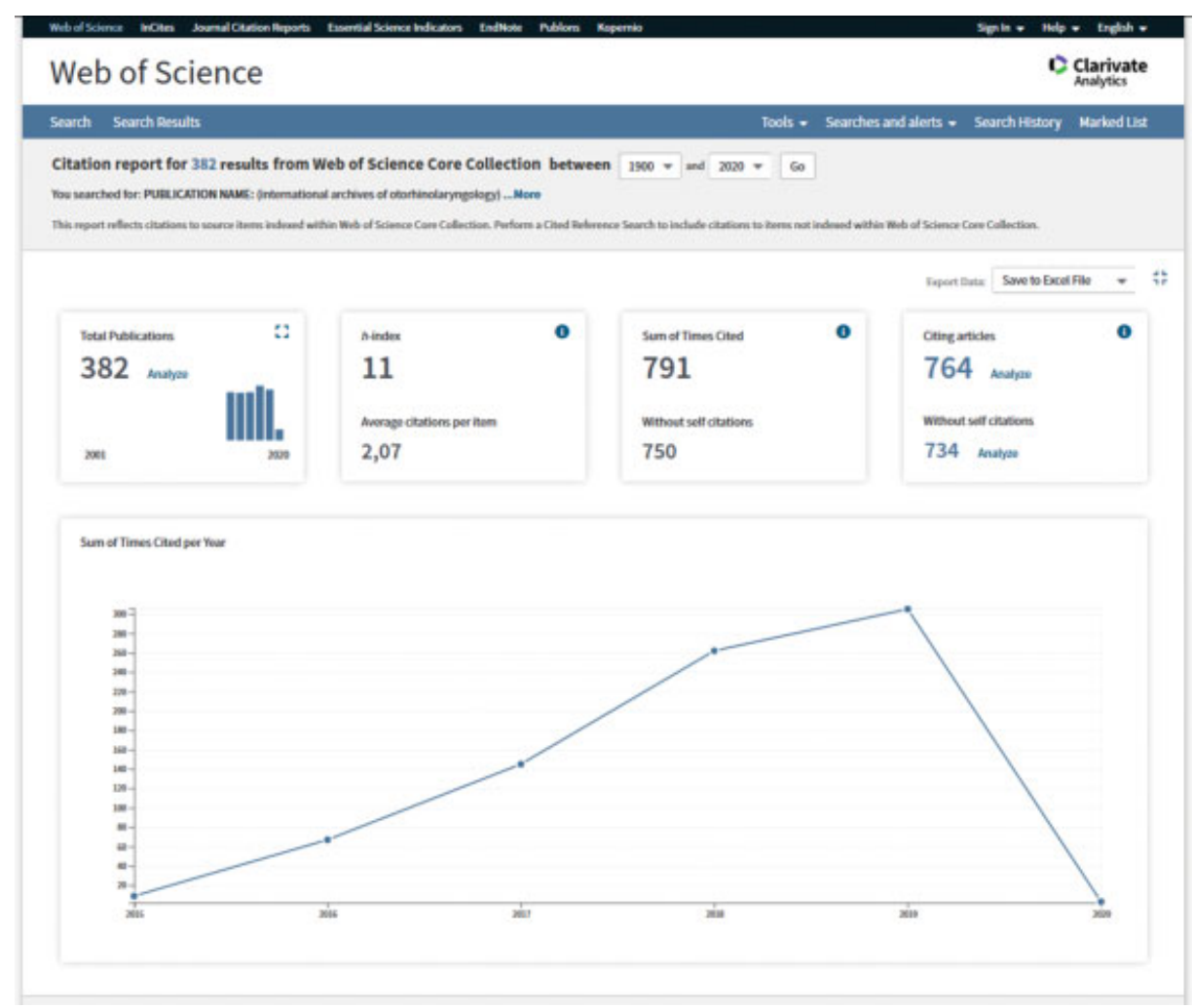

Fig. 2 Citation report for the $I A O$ in the Web of Science.

addition, articles indexed by the ESCI are included in the author's $h$-index calculation and are also analyzed by InCites WoS. ${ }^{3,4}$ With this indexing, the IAO is now monitored by Clarivate Analytics (formerly Thomson Reuters), which uses citation activities to select journals that will receive an official Impact Factor (2).

On November 28, 2019, using the WoS, we found that from 2015 to 2020, the IAO had 382 indexed documents, with an average of 2.07 citations per article (-Fig. 2 and -Table 1). Compared with the average number of citations received ( - Table 1 ), a significant increase in the citations received for review articles is evident. This demonstrates, as in other studies, that reviews receive a higher citation average than other document types. ${ }^{5,6}$ Thus, we can now emphasize the importance of the Award-Winning Research - IAO, to stimulate the submission of new review articles. $^{7}$
Updating the data published by Bento (2017), we would like to highlight the global reach of our publication. ${ }^{8}$ The IAO has published articles submitted by authors from 40 countries (-Fig. 3). With this widespread distribution and the steady increase in manuscript submissions, we are proud to announce the arrival of new Section Editors. IAO now has 7 highly qualified editors ( - Table $\mathbf{2}$ ).

While celebrating our inclusion in these indices, we would like to take the opportunity to specifically recognize the "Top 10 " articles. These articles were selected based on their impact on the WoS citations ( - Table 3 ). We would like to thank all of the Authors who have submitted their work for publication. To all of the Editorial Board and Reviewers, who have always been so attentive to the reviews and have provided relevant feedback, thank you very much for your valuable contributions. And to You the Reader, thank you for choosing our journal as a valuable source of research and information.

Table 1 Descriptive statistics for the number of citations received by document type

\begin{tabular}{|l|l|l|l|}
\hline Document type & N Doc & Times Cited & Average citations per item \\
\hline Article & 321 & 558 & 1,738 \\
\hline Correction & 1 & 0 & 0 \\
\hline Editorial & 12 & 4 & 0,333 \\
\hline Letter & 2 & 1 & 0,5 \\
\hline Review & 46 & 228 & 4,956 \\
\hline TOTAL & 382 & 791 & 2,07 \\
\hline
\end{tabular}




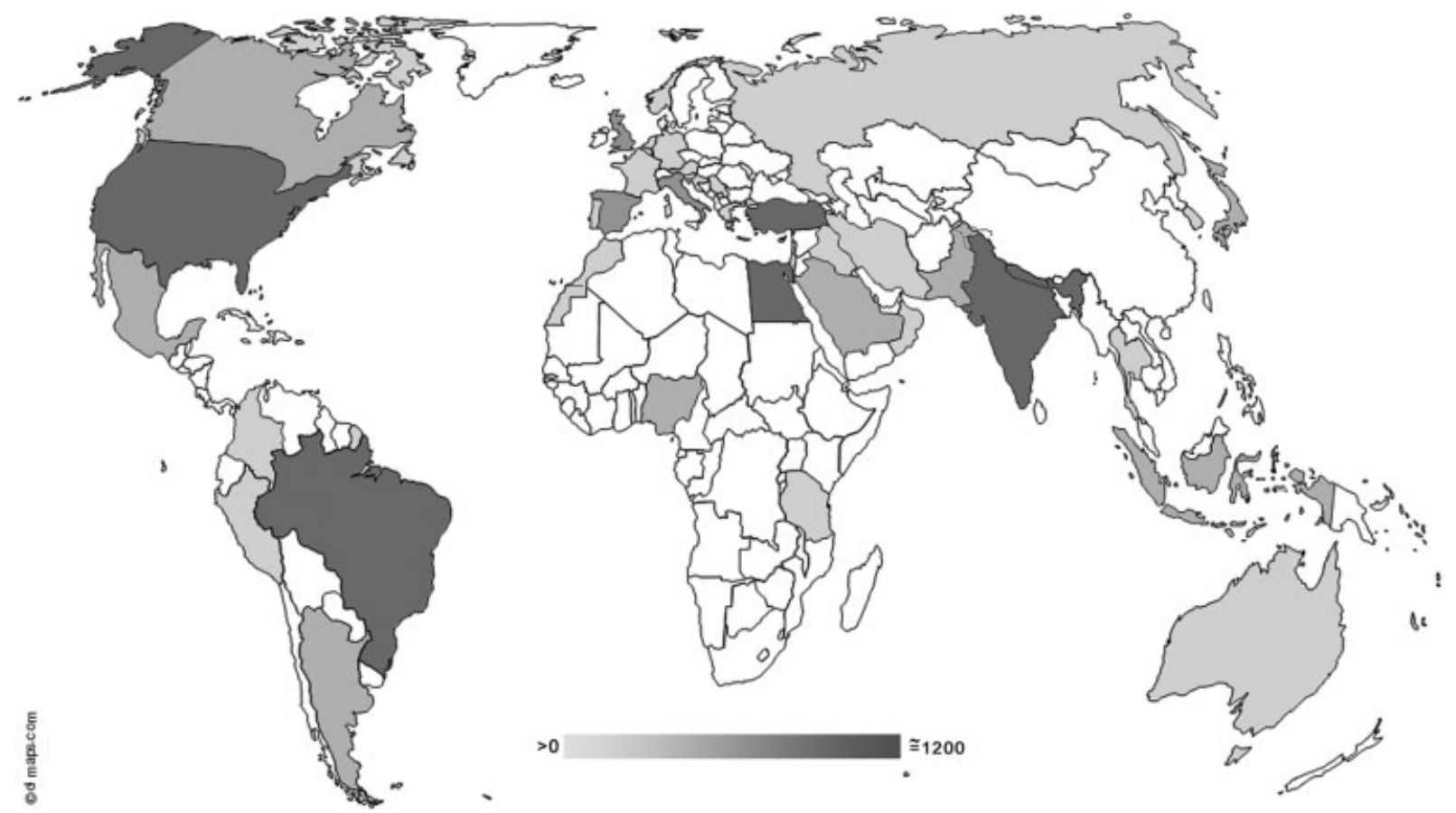

Fig. 3 Distribution of articles published by authors from 40 countries.

Table 2 Section Editors IAO

\begin{tabular}{|l|l|l|}
\hline Section & Name & Institutional Affiliation \\
\hline Ear & Joel Lavinsky & UFRGS, Porto Alegre, Brazil \\
\hline Ear & Roberto Dihl Angeli & ULBRA, Porto Alegre, Brazil \\
\hline Neurotology & Karen de Carvalho Lopes & UNIFESP, São Paulo, Brazil \\
\hline Nose & Roberto Campos Meirelles & UERJ, Rio de Janeiro, Brazil \\
\hline Nose & Thiago Freire Pinto Bezerra & UFPE, Recife, Brazil \\
\hline Throat & Leonardo Haddad & UNIFESP, São Paulo, Brazil \\
\hline Throat & Rui Imamura & USP, São Paulo, Brazil \\
\hline
\end{tabular}

Table 3 Top 10 articles by citation numbers received in Web of Science 2015-2020

\begin{tabular}{|l|l|l|l|l|l|l|l|}
\hline Rank & Title & Authors & $\begin{array}{l}\text { Publication } \\
\text { Year }\end{array}$ & Volume & Issue & DOI & $\begin{array}{l}\text { Total } \\
\text { Citations }\end{array}$ \\
\hline 1 & $\begin{array}{l}\text { Olfaction in } \\
\text { Neurologic and } \\
\text { Neurodegenerative } \\
\text { Diseases: A Litera- } \\
\text { ture Review }\end{array}$ & $\begin{array}{l}\text { Costa Lima Godoy, } \\
\text { Maria Dantas; } \\
\text { Voegels, Richard } \\
\text { Louis; Pinna, Fabio de } \\
\text { Rezende; Imamura, } \\
\text { Rui; Farfel, Jose } \\
\text { Marcelo }\end{array}$ & 2015 & 19 & 2 & $10.1055 /$ s-0034-1390136 & 26 \\
\hline 2 & $\begin{array}{l}\text { Active Bone Conduc- } \\
\text { tion Prosthesis: } \\
\text { Bonebridge (TM) }\end{array}$ & $\begin{array}{l}\text { Zernotti, Mario E.; } \\
\text { Bravo Sarasty, } \\
\text { Andrea }\end{array}$ & 2015 & 19 & 4 & $10.1055 /$ s-0035-1564329 & $\mathbf{2 4}$ \\
\hline 3 & $\begin{array}{l}\text { Cochlear Implanta- } \\
\text { tion and Single-sided } \\
\text { Deafness: A System- } \\
\text { atic Review of the } \\
\text { Literature }\end{array}$ & $\begin{array}{l}\text { Cabral Junior, Fran- } \\
\text { cisco; Pinna, Mariana } \\
\text { Hausen; Alves, } \\
\text { Ricardo Dourado; } \\
\text { dos Santos Malerbi, } \\
\text { Andrea Felice; Bento, } \\
\text { Ricardo Ferreira }\end{array}$ & 2016 & 20 & 1 & $10.1055 / \mathrm{s}-0035-1559586$ & $\mathbf{1 9}$ \\
\hline
\end{tabular}


Table 3 (Continued)

\begin{tabular}{|c|c|c|c|c|c|c|c|}
\hline Rank & Title & Authors & $\begin{array}{l}\text { Publication } \\
\text { Year }\end{array}$ & Volume & Issue & DOI & $\begin{array}{l}\text { Total } \\
\text { Citations }\end{array}$ \\
\hline 4 & $\begin{array}{l}\text { Comorbidities } \\
\text { Associated with } \\
\text { Obstructive Sleep } \\
\text { Apnea: a Retrospec- } \\
\text { tive Study }\end{array}$ & $\begin{array}{l}\text { Pinto, Jose Antonio; } \\
\text { Ribeiro, Davi Knoll; } \\
\text { da Silva Cavallini, } \\
\text { Andre Freitas; } \\
\text { Duarte, Caue; } \\
\text { Freitas, Gabriel } \\
\text { Santos }\end{array}$ & 2016 & 20 & 2 & $10.1055 / \mathrm{s}-0036-1579546$ & 17 \\
\hline 5 & $\begin{array}{l}\text { Tinnitus Neural } \\
\text { Mechanisms and } \\
\text { Structural Changes in } \\
\text { the Brain: The } \\
\text { Contribution of Neu- } \\
\text { roimaging Research }\end{array}$ & $\begin{array}{l}\text { Simonetti, Patricia; } \\
\text { Oiticica, Jeanne }\end{array}$ & 2015 & 19 & 3 & $10.1055 / s-0035-1548671$ & 16 \\
\hline 6 & $\begin{array}{l}\text { Angiosarcoma of the } \\
\text { Head and Neck }\end{array}$ & $\begin{array}{l}\text { Mullins, Brandon; } \\
\text { Hackman, Trevor }\end{array}$ & 2015 & 19 & 3 & $10.1055 / s-0035-1547520$ & 14 \\
\hline 7 & $\begin{array}{l}\text { The Impact of } \\
\text { Dysphagia Therapy } \\
\text { on Quality of Life in } \\
\text { Patients with } \\
\text { Parkinson's Disease } \\
\text { as Measured by the } \\
\text { Swallowing Quality } \\
\text { of Life Questionnaire } \\
\text { (SWALQOL) }\end{array}$ & $\begin{array}{l}\text { Ayres, Annelise; Jotz, } \\
\text { Geraldo Pereira; de } \\
\text { Mello Rieder, Carlos } \\
\text { Roberto; } \\
\text { Schumacher Schuh, } \\
\text { Artur Francisco; } \\
\text { Olchik, Maira } \\
\text { Rozenfeld }\end{array}$ & 2016 & 20 & 3 & $10.1055 / s-0036-1582450$ & 13 \\
\hline 8 & $\begin{array}{l}\text { Foreign Bodies in the } \\
\text { Ear, Nose and Throat: } \\
\text { An Experience in a } \\
\text { Tertiary Care } \\
\text { Hospital in Central } \\
\text { Nepal }\end{array}$ & Parajuli, Ramesh & 2015 & 19 & 2 & $10.1055 / \mathrm{s}-0034-1397336$ & 13 \\
\hline 9 & $\begin{array}{l}\text { Therapeutic Options } \\
\text { in Idiopathic Burning } \\
\text { Mouth Syndrome: } \\
\text { Literature Review }\end{array}$ & $\begin{array}{l}\text { Miziara, Ivan; } \\
\text { Chagury, Azis; } \\
\text { Vargas, Camila; } \\
\text { Freitas, Ludmila; } \\
\text { Mahmoud, Ali }\end{array}$ & 2015 & 19 & 1 & $10.1055 / \mathrm{s}-0034-1378138$ & 13 \\
\hline 10 & $\begin{array}{l}\text { Role of Monocyte } \\
\text { Count and Neutro- } \\
\text { phil-to-Lymphocyte } \\
\text { Ratio in Survival of } \\
\text { Oral Cancer Patients }\end{array}$ & $\begin{array}{l}\text { Bobdey, Saurabh; } \\
\text { Ganesh, Balasubra- } \\
\text { maniam; Mishra, } \\
\text { Prabhashankar; Jain, } \\
\text { Aanchal }\end{array}$ & 2017 & 21 & 1 & $10.1055 / s-0036-1587318$ & 12 \\
\hline
\end{tabular}

\section{References}

1 Simone CB II. Annals of Palliative Medicine has become indexed in Emerging Sources Citation Index Web of Science and Scopus. Ann Palliat Med 2016;5(04):325-326. Doi: 10.21037/apm.2016.10.02

2 Clarivate Analytics. Web of Science Core Collection: journal selection process [Internet]. [cited 2019 Nov 22]. Available at: http://wokinfo.com/media/pdf/ESCI_Fact_Sheet.pdf

3 Taylor \& Francis Group. Emerging Sources Citation Index: An introduction to the ESCI for journal editors [Internet]. [cited 2019 Nov 22]. Available at: https://editorresources.taylorandfrancis.com/understanding-research-metrics/esci/\#

4 Clarivate Analytics. InCites [Internet]. [cited 2019 Nov 28]. Available at: https://incites.clarivate.com/
5 Montefusco AM, Nascimento FP, Sennes LU, Bento RF, Imamura R. Influence of international authorship on citations in Brazilian medical journals: a bibliometric analysis. Scientometrics 2019; 119(03):1487-1496. Doi: 10.1007/s11192-019-03104-0

6 Royle P, Kandala NB, Barnard K, Waugh N. Bibliometrics of systematic reviews: analysis of citation rates and journal impact factors. Syst Rev 2013;2:74. Doi: 10.1186/2046-4053-2-74

7 Jotz GP, Bittencourt AG, Montefusco AM. Award-Winning Research - IAO 2019. Int Arch Otorhinolaryngol 2019;23(04): e373-e374. Doi: 10.1055/s-0039-1700504

8 Bento RF. International Archives of Otorhinolaryngology: 20 Years of Excellence!. Int Arch Otorhinolaryngol 2017;21(01):1-3. Doi: 10.1055/s-0036-1596039 